\title{
Editorial
}

\section{Managing Attention Deficit Hyperactivity Disorder during COVID-19 Pandemic}

\author{
Anjana Rao Kavoor ${ }^{1}$ Sayantanava Mitra² \\ ${ }^{1}$ Mental Health Program, Monash Health, Melbourne, Australia \\ and Faculty of Medicine, University of Queensland Rural Clinical \\ School, Rockhampton, Queensland, Australia \\ ${ }^{2}$ Mental Health Program, Monash Health and Department of \\ Psychiatry, Monash Health, School of Clinical Sciences, Monash \\ University, Melbourne, Australia
}

| Neurosci Rural Pract:2021;12:1-2

Coronavirus disease 2019 (COVID-19) has affected all individuals pervasively. While for some the effects may be mild, for others they have been significant. One such vulnerable population is children suffering from attention deficit hyperactivity disorder (ADHD), which is known to affect more than $10 \%$ of the primary school children. ${ }^{1}$

A meta-analysis has shown that parents of children with ADHD experience significantly more parenting stress than parents of children without ADHD; hyperactive-impulsive symptoms being more predictive of such stress. ${ }^{2}$ These symptoms can be better understood as difficulty in controlling a behavioral response to a given situation or stimulus, manifesting a disruption of the cognitive functions with which most of us refrain ourselves from acting on an impulse. ${ }^{3}$ Keeping the child engaged in activities even while the child is on regular medications, that is, maintaining a routine and structure, and preferably in a familiar environment, forms an important cornerstone of the management of ADHD.

The core features of ADHD might be particularly disruptive during a pandemic like COVID-19. The requirements for physical distancing and hygiene could be difficult to abide by these children, further complicated by their age-commensurate cognitive abilities. Such interactions emphasize the need to assertively treat the symptoms, which is often compromised by the restrictions imposed by lockdowns. Given this unfortunate barrier to the accessibility of timely treatment and continuity of care and increased psychosocial stress faced by the family as a unit, it is of utmost importance to break the vicious cycle by finding alternative (and innovative) ways of service delivery.

While one may presume worsening of disruptive behaviors during lockdown due to restrictions, the results have been mixed. A survey in France, conducted almost a month into lockdown, included 533 parents of children with ADHD

\section{Address for correspondence} Sayantanava Mitra, MD, 246, Clayton Road, Clayton 3168, Melbourne, Australia (e-mail: sayantanava@gmail.com). and found that the sample was split into roughly three equal groups, where $30.96 \%$ of the children were doing better, $34.71 \%$ were worse and the rest maintained status quo in their condition. While improvement in the child's anxiety, less school-related strain, flexible schedules, lesser exposure of their children to negative feedback, and improved self-esteem could explain a good outcome in more than twothirds of their sample; the authors also found influences of optimal lockdown life conditions like sufficient space at home to possibly mediate some of these outcomes. ${ }^{4}$

The paper titled "impact of COVID-19 and lockdown on children with ADHD and their families: an online survey and a continuity care model" ${ }^{5}$ has shed light on the impact of lockdown on individual symptoms of ADHD in children in an Indian context, and performed a brief text-message based intervention to assess feasibility of such an intervention. Among 48 parents who participated, half of the children were found to have worsening of ADHD symptoms with slight or marked increase in the activity level (50.1\%), irritability (45.8\%), and disturbing or disruptive behavior (47.9\%). They also found that the lockdown was "associated with certain positive outcomes," as reflected in about half of the children spending more time with studies, engaging in creative activities and helping out their parents with household chores. The authors found that their text-based intervention was a "feasible" alternative to usual care under the changed circumstances. The findings of this study add to the overall pool of data and informs best practice in ADHD management during this pandemic.

Although the current paper had a lower sample size-and therefore a lower power-than the study by Bobo et al, ${ }^{4}$ it has used more precise questions by adapting a scale for individual symptoms of ADHD. The use of a text-message based intervention incorporating techniques like contingency management and positive parenting principles are further (c) 2021. Association for Helping Neurosurgical Sick People.

This is an open access article published by Thieme under the terms of the Creative Commons Attribution-NonDerivative-NonCommercial-License, permitting copying and reproduction so long as the original work is given appropriate credit. Contents may not be used for commercial purposes, or adapted, remixed, transformed or built upon. (https://creativecommons.org/licenses/by-nc-nd/4.0/)

Thieme Medical and Scientific Publishers Pvt. Ltd. A-12, 2nd Floor, Sector 2, Noida-201301 UP, India 
improvements. The latter is in keeping with the recommendations of the European ADHD guidelines group, which advocates for the central role of behavioral parenting training strategies in the management of ADHD, given its positive effects on parenting and effectiveness in reducing oppositional, defiant, and disruptive behaviors. ${ }^{6}$

In an effort to investigate the benefits of self-help interventions in ADHD, Dose et $\mathrm{al}^{7}$ set a precedence to the current study where they found telephone-assisted self-help parenting behavioral intervention to enhance effects of methylphenidate treatment in families who completed the intervention. The text-message based intervention used by the current authors ${ }^{5}$ are in keeping with recent practice adaptations to continue relevant mental health-related services via telephone or appropriate alternative technology. Using a text-based intervention may be more suitable for families who are not so well versed with technology since it only entails reading/viewing media messages or videos, especially in a country like India, with unreliable data connectivity. As noted earlier, the authors have found a reasonable level of the satisfaction of parents/families, which instills confidence in this method.

Since the effectiveness of telepsychiatry has not been contrasted with face-to-face interventions, it calls for more research in this area, especially considering the high acuity of needs during this pandemic. This study has the potential to stimulate further research in this regard and help in developing a more structured multipronged intervention.

\section{Funding}

None.

\section{Conflict of Interest}

None declared.

\section{References}

1 Venkata JA, Panicker AS. Prevalence of attention deficit hyperactivity disorder in primary school children. Indian J Psychiatry 2013;55(4):338-342

2 Theule J, Wiener J, Tannock R, et al. Parenting stress in families of children with ADHD: a meta-analysis. J Emot Behav Disord 2013;21(1):3-17

3 Barkley RA, Understanding AD/HD and self-control: social and clinical implications. In: ADHD and the Nature of Self Control. 1997; New York: Guilford

4 Bobo E, Lin L, Acquaviva E, et al. How do children and adolescents with Attention Deficit Hyperactivity Disorder (ADHD) experience lockdown during the COVID-19 outbreak?. Encephale 2020;46(3S):S85-S92

5 Shah R, Venkatesh Raju V, Sharma A, Grover S. Impact of COVID-19 and lockdown on children with ADHD and their families: an online survey and a continuity care model. J Neurosci Rural Pract 2021;12 (1):71-79

6 Cortese S, Asherson P, Sonuga-Barke E, et al; European ADHD Guidelines Group. ADHD management during the COVID-19 pandemic: guidance from the European ADHD Guidelines Group. Lancet Child Adolesc Health 2020;4(6): 412-414

7 Dose C, Hautmann C, Buerger M, Schuermann S, Woitecki K, Doepfner M. Telephone-assisted self-help for parents of children with attention-deficit/hyperactivity disorder who have residual functional impairment despite methylphenidate treatment: a randomized controlled trial. J Child Psychol Psychiatry 2017;58(6):682-690 